\title{
Vibrotactile adaptation on the face
}

\author{
MARK HOLLINS, KIMBERLY A. DELEMOS, and ALAN K. GOBLE \\ University of North Carolina, Chapel Hill, North Carolina
}

\begin{abstract}
Threshold amplitude for vibration is elevated if testing is preceded by extended exposure to a vibratory adapting stimulus of appropriate amplitude and frequency. This phenomenon, previously studied almost exclusively on the hand, is here shown for the first time to occur on the face as well. Adaptation is then used analytically to determine that the two-branched thresholdversus-frequency function obtained on the face by Verrillo and Ecker (1977) represents the activity of two distinct mechanisms. Action spectra of vibrotactile adaptation reveal the presence of both mechanisms even in subjects whose unadapted threshold function (like that reported by Barlow, 1987) shows no sign of duplexity. Finally, the data suggest that on the face (unlike the hand), cross-channel adaptation may occur at high adapting amplitudes.
\end{abstract}

It has been known for half a century that the ability to detect vibratory stimulation of the skin is extremely susceptible to adaptation (Cohen \& Lindley, 1938; Wedell \& Cummings, 1938), and evidence from a number of studies indicates that this vibrotactile adaptation occurs independently within Pacinian and non-Pacinian systems (Gescheider, Frisina, \& Verrillo, 1979; Gescheider \& Verrillo, 1979; Hahn, 1968; Verrillo \& Gescheider, 1977). Furthermore, recent work on a particular mechanoreceptive channel (NP I of Bolanowski, Gescheider, Verrillo, \& Checkosky, 1988) has shown that adapting stimuli of different frequencies will elevate the channel's threshold by comparable amounts if their amplitudes, expressed in decibels SL for the channel, are the same (Hollins, Goble, Whitsel, \& Tommerdahl, 1990); thus, an action spectrum of adaptation for the channel-that is, a function showing the amplitudes needed at different adapting frequencies to raise the threshold of the channel (measured at a fixed frequency) by a criterion amountparallels its threshold-versus-frequency function. This result suggests that the determination of action spectra may constitute a method of identifying mechanoreceptive channels that are present, yet not contributing measurably to the unadapted threshold function.

One constraint on our present understanding of vibrotactile adaptation is that it is based almost entirely on measurements made on the hand or fingertip. In the present study, we sought to remove this limitation by testing a differently innervated region, the skin of the lower face. Anatomical study of the perioral region indicates a number of differences between its sensory innervation and that of the hand, the most salient of which is an apparent lack

This research was supported by U.S. Public Health Service Grant DE-07509, and by a National Science Foundation Graduate Fellowship to K. A. Delemos. The authors are indebted to Barry Whitsel for valuable discussion, and to Mark Tommerdahl for expert technical assistance. Correspondence should be addressed to Mark Hollins at the Department of Psychology, C.B. 3270, Davie Hall, University of North Carolina, Chapel Hill, NC 27599. of Pacinian corpuscles in the face (Darian-Smith, 1973; Halata \& Munger, 1983; Munger \& Halata, 1983). Singlefiber recordings from the inferior alveolar and infraorbital nerves likewise reveal no evidence of Pacinian afferents (Johansson \& Olsson, 1976; Johansson, Trulsson, Olsson, $\&$ Westberg, 1988). If vibrotactile adaptation were found to occur in a tissue so different from the glabrous skin of the hand, and to obey rules (e.g., channel independence) already known to apply on the hand, this would substantially extend the generality of our present understanding of the vibration sense.

We also hoped that adaptation techniques could help us to resolve a seeming discrepancy in the literature between two sets of measurements of vibrotactile thresholds in the orofacial region. Verrillo and Ecker (1977), in the course of a study of tic douloureux, examined the facial vibrotactile sensitivity of a control group of neurologically normal subjects, and found it to be a two-branched function of frequency. Threshold was essentially independent of frequency below $40 \mathrm{~Hz}$, but at higher frequencies, threshold began to decline at a rate of about $6 \mathrm{~dB} /$ octave, reaching a minimum in the neighborhood of $200 \mathrm{~Hz}$. Verrillo and Ecker suggested that the U-shaped high frequency branch of the threshold function might reflect the activity of Golgi-Mazzoni corpuscles. Barlow (1987) also measured vibrotactile thresholds on the face, but with a different result: a smooth, unbroken function that declined only slightly as frequency rose. Not until frequency exceeded $400 \mathrm{~Hz}$ did threshold begin to drop abruptly. There is no sign in Barlow's shallow function of Verrillo and Ecker's (1977) U-shaped branch.

This difference in results is probably not a result of the spatial or temporal parameters of the stimulus, for these were similar in the two studies. Specific location on the face is also an unlikely explanation, because Barlow made measurements at a number of locations and found them to yield comparable results. Intersubject variability is a remaining possibility, which we hoped to explore in the present study by closely comparing the results of individual observers. Despite the difference in results, it 
is important to note that neither Verrillo and Ecker (1977) nor Barlow (1987) found any evidence of Pacinian involvement in vibrotactile sensitivity on the face.

The present study involved three experiments. In the first, threshold-versus-frequency functions were obtained, in order to learn whether any (or all) of our subjects would give evidence of a two-branched curve. Two sizes of contactor were used in this experiment, to explore the possibility that one of the channels might show greater spatial summation than the other; this technique has been extremely valuable in research on the hand and arm, because of the spatial summation evinced by the Pacinian channel (Gescheider, 1976; Merzenich \& Harrington, 1969; Verrillo, 1963, 1968).

The primary purpose of the second experiment was to determine whether vibrotactile adaptation occurs on the face. This question was answered in the affirmative. Measurements were made with a $50-\mathrm{Hz}$ test stimulus, this being approximately the frequency at which, in Verrillo and Ecker's (1977) data, the high- and low-frequency branches of the threshold function intersect. We compared the threshold-elevating effectiveness of two adapting stimuli, differing in frequency ( 10 and $50 \mathrm{~Hz}$ ) but equated in sensation level (17 dB SL), to look for evidence of more than one mechanism. If the two branches of Verrillo and Ecker's function represent separate channels, we might expect, by analogy with data obtained on the hand (Hollins et al., 1990), that threshold would be raised more by an adapter to which both channels are sensitive $(50 \mathrm{~Hz})$ than by one $(10 \mathrm{~Hz})$ that desensitizes only a single channel.

In the third experiment, two action spectra of vibrotactile adaptation were obtained. One consisted of those adapting amplitudes, across a range of frequencies from 10 to $150 \mathrm{~Hz}$, that were needed to elevate vibrotactile threshold at $10 \mathrm{~Hz}$ by $10 \mathrm{~dB}$; the other consisted of analogous measurements made with a test frequency of $150 \mathrm{~Hz}$. We hoped by means of this experiment to answer three questions: (1) whether the two branches that constitute the unadapted threshold function of some subjects reflect the activity of separate mechanisms; (2) whether these two mechanisms are present even in a subject whose unadapted threshold function gives no indication of duplexity; and (3) whether vibrotactile mechanisms in the face obey the principle of channel independence.

\section{EXPERIMENT 1}

\section{Method}

Apparatus. In all the experiments in this study, vibratory stimuli were delivered to the subjects by a Chubbuck (1966) small motion biological stimulator, interfaced with a custom-built Cantek 500 controller. This system's feedback circuitry allowed the amplitude of stimulation to be held constant at any desired level within its range, independent of moderate variations in the mechanical resistance offered by the skin. The sinusoidal voltage used to drive the transducer was provided by a Wavetek Model 182A function generator, the frequency output of which was directly controlled (via parallel port and digital-to-analog converter) from a PDP-11/02 computer. The computer also gated this sinusoidal signal, and controlled its amplitude by means of a voltage divider. Fast Fourier transforms of displacement readout, and visual inspection of waveforms on an oscilloscope, showed that onset and offset transients were negligible.

The contactor was a cylinder of Delrin polyacetal thermoplastic, milled flat at the end. Contactors of different sizes could be substituted for one another, to change the size of the area of skin stimulated. A flat brass ring, $25.4 \mathrm{~mm}$ in outer diameter and $3.0 \mathrm{~mm}$ in thickness, was applied to the skin surrounding the area to be stimulated, in order to reduce the lateral spread of vibration (Verrillo, 1968). The ring was brought into increasing contact with the slightly convex surface of the cheek until contact was just visible all around its outer rim. Rings differing in inner diameter were used in conjunction with the differently sized contactors, so that there was always a 1-mm gap between the edge of the contactor and the inner edge of the ring.

Subjects. The three authors, ages 43,22 , and 23 , respectively, served as the subjects in all the experiments. The 2 male subjects shaved several hours before a session.

Procedure. The subject sat facing a table to which a mill mount was rigidly attached. An individualized bite bar, made of dental impression compound surrounding a Y-shaped aluminum core, was affixed to the mill mount in order to stabilize the head. Once the subject was comfortably positioned, the brass ring (attached to the table by Flexaframe scaffolding) was brought into contact with the skin of the cheek, its center located approximately $2 \mathrm{~cm}$ lateral to the corner of the mouth. Next, the transducer was oriented perpendicular to the skin surface (i.e., roughly horizontally), and positioned so that the contactor was centered within the brass ring. The transducer was mounted on an adjustable boom that was not in contact with the table.

The contactor was advanced toward the skin, by increasing the DC voltage delivered to the transducer, until the force readout on the controller indicated that the contactor had encountered nonzero mechanical resistance. An opposing force of $0.1 \mathrm{~g}$ could be detected in this way. In our earlier study, which involved stimulation of the fingertip, this criterion of skin contact had correlated closely with the subject's verbal report of a tactile sensation (Hollins et al., 1990); but in the present research, initial contact defined by force measurement was typically insufficient to produce a threshold sensation. This observation is consistent with our sensitivity measurements, which showed vibrotactile threshold on the region of cheek studied to be higher than that obtained by Hollins et al. (1990) on the same subjects' index fingerpads. Once contact had been established, the contactor was advanced a further 500 microns into the skin.

In all three experiments, computer-controlled Békésy tracking was used to determine threshold for test stimuli, which consisted of 500 -msec bursts of vibration, delivered at the rate of $1 / \mathrm{sec}$. If the subject indicated detection of a stimulus, by operating a pushbutton during its occurrence or during the 500 -msec interstimulus interval that followed, the next stimulus was lowered in amplitude by $1 \mathrm{~dB}$. When a stimulus went undetected, however, the amplitude on the following trial was increased by $1 \mathrm{~dB}$. Consequently, the amplitude of the stimulus approached and then hovered about a just-detectable level.

In this first experiment, thresholds were determined at 20 frequencies (ranging from 10 to $200 \mathrm{~Hz}$ in 10- $\mathrm{Hz}$ steps) and two contactor sizes ( 2 and $8 \mathrm{~mm}$ in diameter). Trials were arranged in series of 50 , called test periods, within which frequency and contactor size remained fixed. Test periods, in turn, were arranged in blocks of 20 , each frequency being represented once per block. Frequencies were randomly ordered within a block, a different random series being used for each block. Ten-second pauses separated consecutive test periods within a block, and breaks of at least $15 \mathrm{~min}$ were given between blocks. Contactor size remained the same throughout a block, but alternated randomly (without replacement) 
across blocks. Daily experimental sessions usually consisted of 3 or 4 blocks. In all, each subject participated in 10 blocks, 5 for each contactor size.

\section{Results and Discussion}

The first step in the analysis of data was the conversion of stimulus amplitudes to decibel values (re: $1-\mu \mathrm{m}$ displacement), which were used in all subsequent calculations. The value of each crossover point, defined as the mean of the two test stimulus values bracketing a transition from "yes" to "no" responding, or the reverse, was determined, and all crossover points within a test period were averaged to give a measure of threshold for that period.

The resulting threshold values are shown in Figure 1, with separate panels illustrating the data for the 3 subjects. Data obtained with the 2- and 8-mm contactors are plotted with open and filled symbols, respectively.

At low frequencies, the threshold functions of the 3 subjects are similar, declining gradually as frequency increases along a curve resembling that of the NP I mechanism on the hand (Bolanowski et al., 1988). At high frequencies, however, there are marked differences among the 3 subjects. Subject M's function shows a clear break at $60 \mathrm{~Hz}$, beyond which threshold declines steadily at a rate of about $6 \mathrm{~dB} /$ octave, equivalent to the slope of the high-frequency mechanism discovered on the face by Verrillo and Ecker (1977). Subject K's data, however, show no such discontinuity: They level off at high frequencies, thus resembling Barlow's (1987) threshold measurements. Subject A's data follow an intermediate course, tending slightly downward at frequencies above $60 \mathrm{~Hz}$.

The results thus establish that there are substantial individual differences in the threshold function on the face-differences comparable to those separating the group functions of Verrillo and Ecker (1977) and Barlow (1987). We will consider in the General Discussion one possible reason for these differences.

Another noteworthy feature of the data in Figure 1 is that, for each subject, the threshold function obtained with the 8-mm contactor parallels that obtained with the 2-mm contactor. For Subject $M$, the functions are separated by about $1 \mathrm{~dB}$, while the separations are larger for the other 2 subjects. This effect of contactor size may represent a modest amount of spatial summation, or, alternatively, a difference in the criteria subjects used in the two experimental conditions. However, the separation of the curves does not change systematically as a function of frequency, as would be expected if two mechanisms differing in their spatial integration characteristics determined threshold at low and high frequencies, respectively. Specifically, the lack of separation of the two functions in the case of Subject $\mathbf{M}$ makes clear that Pacinian corpuscles, which show marked spatial summation (Bolanowski et al., 1988; Verrillo, 1968), are not the receptor system underlying the high-frequency limb of his threshold functions-a conclusion that is further borne out by the slope of this branch ( $-6 \mathrm{~dB} /$ octave), which is only half as steep as that of the Pacinian system (Verrillo, 1966). This lack of evidence of Pacinian involvement for any subject confirms Barlow (1987) and Verrillo and Ecker (1977).

Neither the data of Experiment 1, nor those of earlier studies, resolve the question of whether multiple mechanisms underlie the threshold function. The data of the different subjects may simply reflect the presence of a single mechanism, the frequency characteristic of which is complex, and varies from one individual to another. We used vibrotactile adaptation as a tool to address this question in subsequent experiments.

\section{EXPERIMENT 2}

This experiment was designed to reveal whether vibrotactile adaptation can occur on the face, and if so, how that process compares with adaptation on the finger and hand, as measured in earlier studies.

\section{Method}

The subjects, apparatus, and set-up phase of the procedure were the same as in Experiment 1, but the protocol was modified to incorporate periods of adaptation as well as test periods. A suprathreshold adapting stimulus was presented repeatedly over the course of an 8-min segment of the run, and its effect on threshold was monitored. The subject was exposed to this adapting stimulus for $49.5 \mathrm{sec}$ out of every minute, the remaining $10.5 \mathrm{sec}$ being reserved for a test period during which Bekésy tracking was used to measure threshold. A test period contained 10 trials, the first of which began $0.5 \mathrm{sec}$ after the offset of the adapting stimulus; as in Experiment 1 , each trial consisted of a 500 -msec burst of vibration followed by a 500 -msec period of no stimulation.

This 8-min adaptation phase of each run was preceded and followed by segments (the baseline phase and recovery phase, respectively) during which threshold was periodically measured, but no adapting stimulus was presented. As in the adaptation phase, test stimuli during the baseline and recovery phases were presented only during the final $10 \mathrm{sec}$ of each minute.

The test stimulus frequency was $50 \mathrm{~Hz}$ throughout. We reasoned that, by comparing the effects produced by different adapting stimuli on threshold at this test frequency, which is close to the break in Verrillo and Ecker's (1977) threshold function, we might uncover evidence that multiple mechanisms contribute to detection. Two adapting frequencies, 10 and $50 \mathrm{~Hz}$, were employed in separate runs; both adapters were set at $17 \mathrm{~dB} \mathrm{SL}$ with respect to the thresholds determined in Experiment 1 . In addition, control runs were carried out in which no adapting stimulus was presented. Runs of the three types were ordered randomly without replacement. In all, five runs of each type were carried out on each subject. All stimuli in Experiments 2 and 3 were delivered with the 8-mm contactor.

\section{Results and Discussion}

The results are shown in Figure 2. Data obtained with the 10 - and $50-\mathrm{Hz}$ adapting stimuli are shown by the filled and open circles, respectively. Each point plots the threshold for a particular test period within the run: The first 3 points, plotted on the left, show thresholds obtained during the three baseline periods; the next 8 points represent test periods that immediately followed 50 -sec bouts of adaptation; and the last 8 points are the results from the recovery phase, during which the adapting stimulus was discontinued. The subjects participated in five runs under each adapting condition, so each point in the figure 


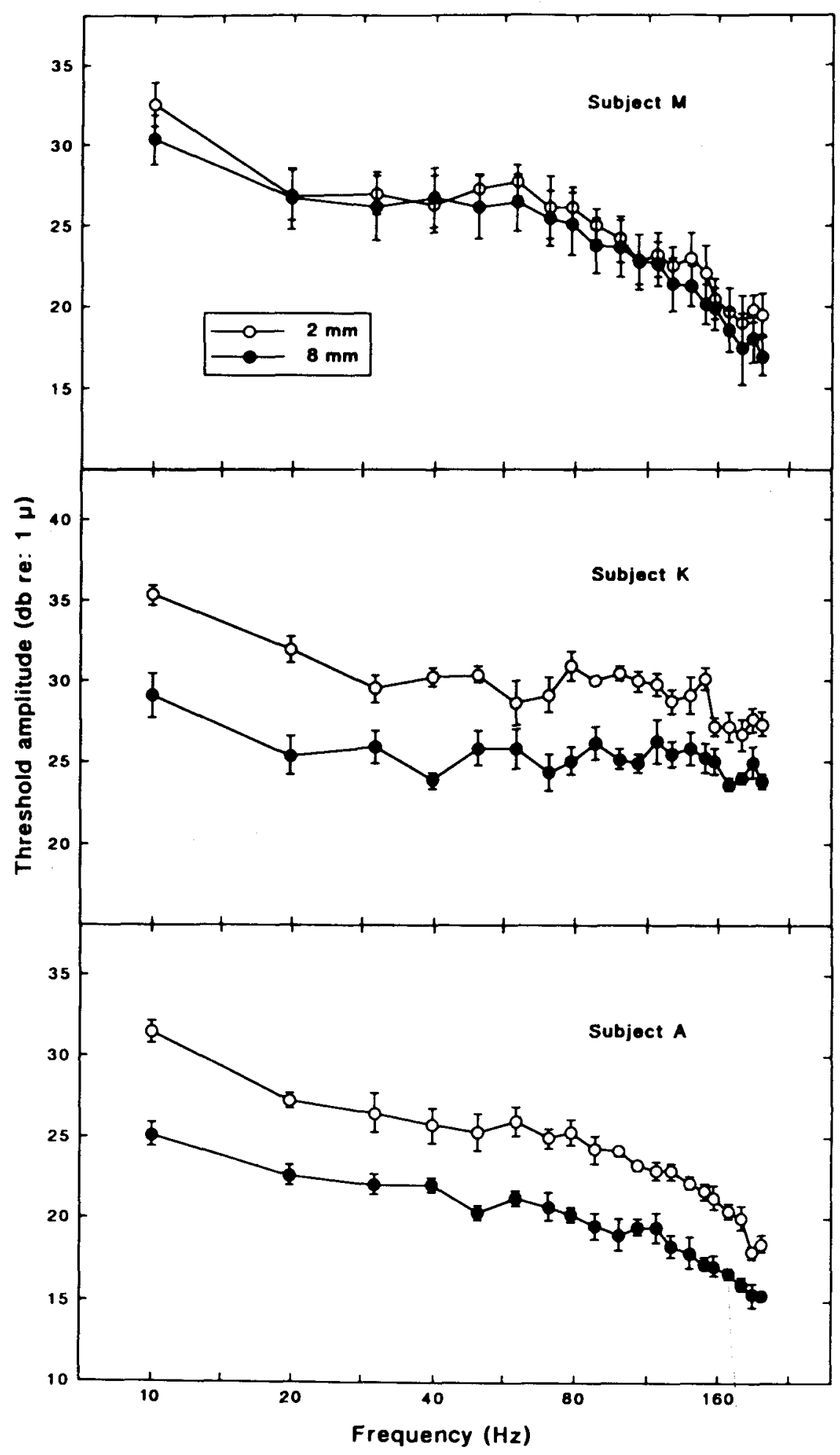

Figure 1. Vibrotactile threshold amplitude as a function of frequency and contactor size. Amplitude is expressed in decibels re: 1 micron peak displacement. Measurements obtained with contactors of 2- and 8-mm diameter are shown by open and filled symbols, respectively; error bars indicate 1 SEM above and below each point, which is the mean of five determinations. 


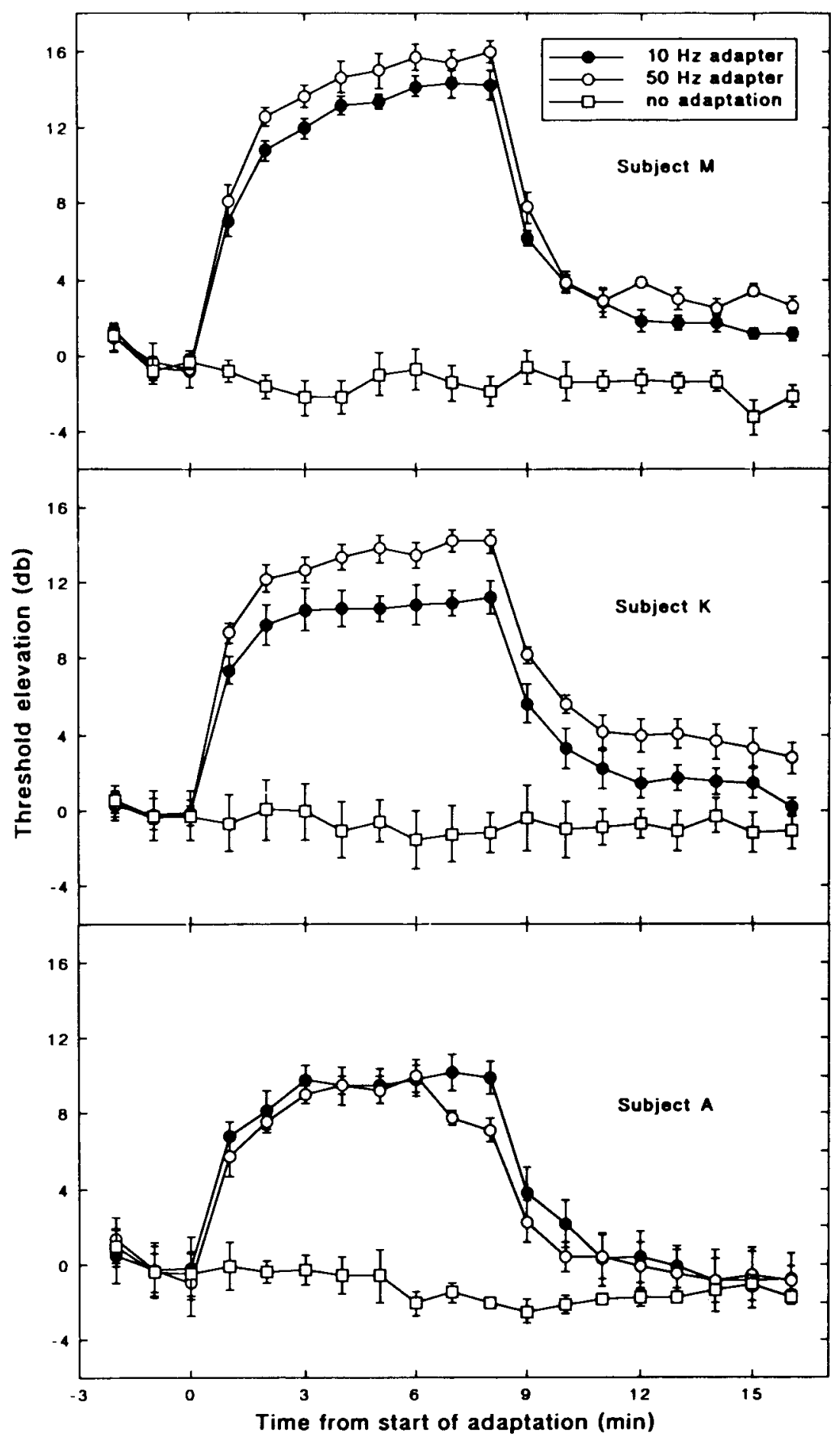

Figure 2. Changes in vibrotactile threshold during adaptation and recovery, measured with a 50-Hz test stimulus. The frequency of the adapting stimulus was either $10 \mathrm{~Hz}$ (filled circles) or $50 \mathrm{~Hz}$ (open circles). Adaptation began immediately following the third determination of threshold within a run, and continued (with brief interruptions for threshold measurement) for $8 \mathrm{~min}$; recovery was then monitored for the next $8 \mathrm{~min}$. Squares plot the results of control runs in which no adapting stimulus was presented. Threshold is expressed in decibels relative to its mean value during the baseline period. Error bars indicate 1 SEM above and below each point, which is the mean of five determinations. 
is the mean of five independent determinations. For each data set, the average threshold obtained during the baseline phase was set at zero, and the ordinate indicates changes from this unadapted value.

The most salient result of this experiment is its clear demonstration that vibrotactile adaptation occurs on the face. The phenomenon is therefore not unique to the extremities, the only sites on which it has previously been demonstrated. The present data show that threshold rises abruptly, and then levels off, as exposure to the adapting stimulus proceeds. Following the discontinuance of the adapting stimulus, threshold recovers, again following a negatively accelerated course. For 1 subject (A), threshold returns to its baseline level by the end of the run, but for the other 2 subjects it remains somewhat elevated, a result previously obtained on the finger (Hollins et al., 1990).

Of primary interest are the relative amounts of adaptation produced by the two adapting stimuli. For Subjects $M$ and $A$, the two stimuli have induced roughly equivalent amounts of threshold elevation, while for Subject $\mathrm{K}$, the $50-\mathrm{Hz}$ adapter appears to have been somewhat more effective than the $10-\mathrm{Hz}$ adapter. To test these impressions quantitatively, we calculated an index of adaptation magnitude for each run by averaging the thresholds obtained during the last $3 \mathrm{~min}$ of adaptation, and subtracting from this value the mean threshold measured during the 3-min baseline period preceding adaptation. For each subject, the indices so obtained were compared across adapting stimuli. Statistically, there was no difference between the amounts of threshold elevation produced by the two adapters for either Subject M or Subject A $(p>.1)$, but for Subject $\mathrm{K}$, the difference was significant $[t(8)=$ $3.8, p<.01$, two-tailed].

The difference in effectiveness of the two adapting stimuli in the case of Subject $\mathrm{K}$ suggests that two receptor systems are involved, either of which can mediate detection of the $50-\mathrm{Hz}$ test stimulus. According to this interpretation, the $50-\mathrm{Hz}$ adapter desensitizes both mechanisms, but the $10-\mathrm{Hz}$ adapter leaves one mechanism (the sensitivity of which increases with frequency) relatively unchanged, and therefore produces less of a rise in behavioral threshold. The results are surprising, in that $\mathrm{K}$ is the one subject whose unadapted threshold function (Experiment 1) gives no indication of duplexity.

These puzzling findings led us to conduct a third experiment, with which we hoped to answer two questions: (1) Do the two branches of the threshold function obtained in Experiment 1 (and by Verrillo and Ecker, 1977) actually reflect the presence of two independent mechanisms? (2) Are both of these mechanisms present even in individuals, like Subject K and Barlow's (1987) subjects, whose threshold function shows no inflection?

\section{EXPERIMENT 3}

The goal of this experiment was to obtain information about the mechanisms underlying vibrotactile adaptation, by determining action spectra for the adaptation process.
Such action spectra indicate the adapting amplitudes needed, at each of a variety of adapting frequencies, to raise the threshold of the test stimulus by a criterion amount ( $10 \mathrm{~dB}$ in this case). Two separate action spectra were determined, using test frequencies of 10 and $150 \mathrm{~Hz}$, respectively. It was anticipated that a comparison of these two action spectra would provide strong evidence that vibrotactile threshold on the face, over this frequency range, is mediated either by a single mechanism (if the action spectra matched) or by multiple mechanisms (if the spectra were different).

\section{Method}

A series of runs, similar in temporal structure to those of Experiment 2, was needed to determine each point on an action spectrum. The process began, for a particular combination of test and adapting frequencies, with a run in which the adapting stimulus was set at an amplitude between 10 and $20 \mathrm{~dB}$ SL. The amount of threshold elevation that occurred was noted and was used to guide the choice of adapting amplitude for the next run. Relying on the fact that, for a given combination of test and adapting frequencies, increases in the amplitude of the adapting stimulus generally lead to greater amounts of threshold elevation, the experimenter increased the adapting amplitude by several decibels if the initial run had involved less than $10 \mathrm{~dB}$ of threshold elevation, or lowered the adapting amplitude if more than $10 \mathrm{~dB}$ of threshold elevation had been produced. This process continued until a pair of runs were obtained that bracketed $10 \mathrm{~dB}$ of adaptation. The entire process (using a new set of adapting amplitudes, but the same test and adapting frequencies) was repeated in a later session, and data from the two runs were then combined into a single series. Interpolation within this series was used to estimate the adapting amplitude that would produce exactly the criterion amount of threshold elevation. Critical adapting amplitudes were determined in this way for adapting frequencies ranging from 10 to $150 \mathrm{~Hz}$ in steps of $20 \mathrm{~Hz}$, for each of the two test frequencies. Blocks of runs with $10-$ and $150-\mathrm{Hz}$ test stimuli were interspersed.

At frequencies above $100 \mathrm{~Hz}$, high-amplitude stimuli produced audible sounds. To mask these, pulsating broad-band noise was presented through earphones to the subject, throughout Experiment 3. The subjects adjusted the intensity of the noise to a comfortable but effective level. In some situations, a noise masker can elevate vibrotactile thresholds (Gescheider \& Niblette, 1967); such cross-modal interaction was apparently not a factor in the present study, however, as is evinced by the fact that in Experiment 3, in which an auditory masker was used, mean unadapted thresholds at 10 and $150 \mathrm{~Hz}$ (respectively 26 and $23 \mathrm{~dB}$ re: $1-\mu \mathrm{m}$ displacement) were comparable to analogous measurements made several months earlier in Experiment 1, without an auditory masker (28 and $21 \mathrm{~dB})$.

\section{Results and Discussion}

Two representative sets of measurements are shown in Figure 3. These data indicate the elevation of Subject K's threshold for a $150-\mathrm{Hz}$ test stimulus, following adaptation at either $30 \mathrm{~Hz}$ (filled symbols) or $130 \mathrm{~Hz}$ (unfilled symbols). For each adapting frequency, threshold elevation is an increasing function of adapting amplitude. The dashed line at a height of $10 \mathrm{~dB}$ threshold elevation represents the criterion we used in determining action spectra. It can be seen that for this subject and test frequency, $130 \mathrm{~Hz}$ was some $12 \mathrm{~dB}$ more effective an adapting frequency than was $30 \mathrm{~Hz}$. 


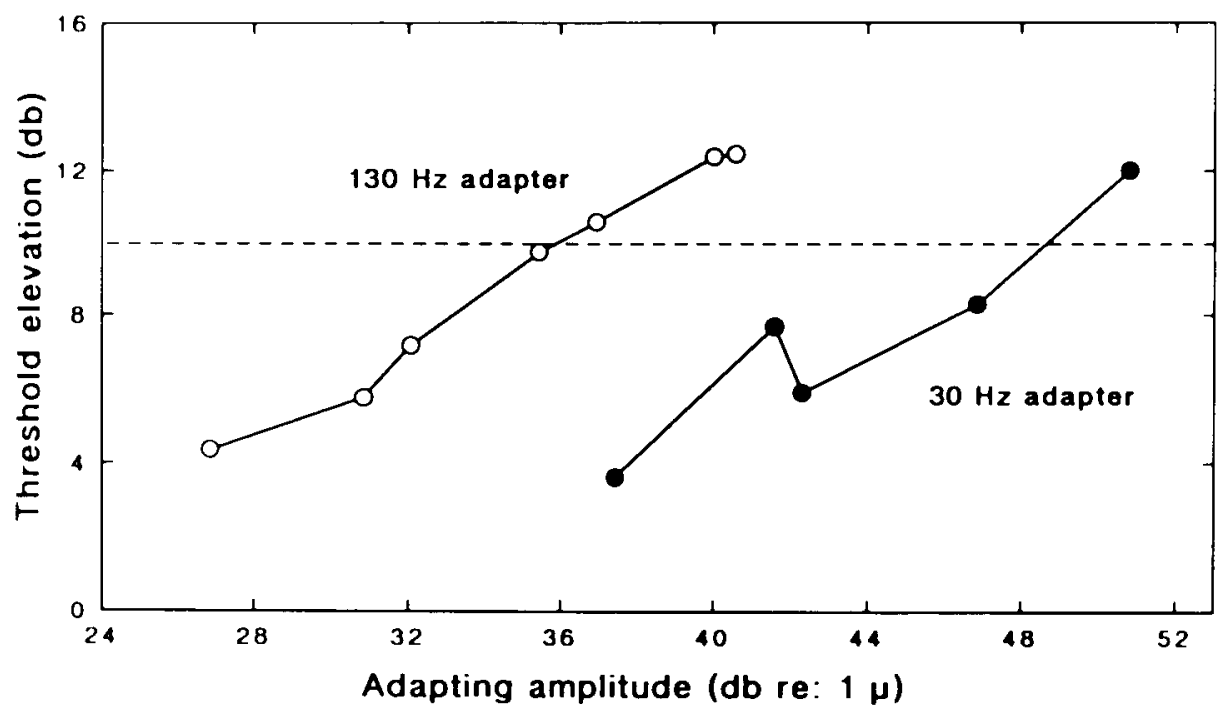

Figure 3. Representative data from one subject $(\mathrm{K})$ showing the elevation of threshold at $150 \mathrm{~Hz}$, produced by $30 \mathrm{~Hz}$ (filled symbols) or $130 \mathrm{~Hz}$ (open symbols) stimuli over a range of amplitudes. Threshold elevation is expressed in decibels above the mean baseline threshold for all runs on this subject in which a 150-Hz test stimulus was used. The dashed horizontal line represents the criterion threshold elevation $(10 \mathrm{~dB})$ used in the determination of action spectra.

The full action spectra are shown in Figure 4. For each subject, results with the $10-\mathrm{Hz}$ test stimuli are plotted with filled symbols, and those for the $150-\mathrm{Hz}$ test stimuli, with unfilled symbols. The 3 subjects' data show similar patterns. For all 3, the function for the $10-\mathrm{Hz}$ test is relatively flat; the $150-\mathrm{Hz}$ action spectrum lies above it in each panel and has a clear inflection at an intermediate frequency $(50-90 \mathrm{~Hz})$.

These results can in large part be understood in terms of two independent mechanoreceptive systems, with frequency characteristics matching the two branches of the threshold function shown by Verrillo and Ecker's (1977) subjects, and by Subject $M$ in Experiment 1 of the present study. Consider first the action spectrum for the $10-\mathrm{Hz}$ test condition. This is relatively flat across frequencies, like the low-frequency portion of the unadapted threshold function, and it invites the interpretation that the same system (let us for simplicity call it the frequency-independent mechanism, although its sensitivity actually changes somewhat as a function of frequency) is determining threshold in both cases. It is entirely reasonable that this channel should be the more sensitive of the two to the test stimulus throughout the $10-\mathrm{Hz}$ action spectrum measurement series, if we assume that the threshold of the other mechanism, the one represented by the high-frequency branch of the unadapted function (let us call it the frequencydependent mechanism) continues to rise as frequency is lowered, at a rate of approximately $6 \mathrm{~dB} /$ octave.

Consider now the more complex action spectrum obtained with $150-\mathrm{Hz}$ test stimuli. The high-frequency branch of this function, with a negative slope of 6-7 dB/octave, appears to represent the activity of the frequency-dependent mechanism. This, too, is what we would expect, under the assumption that an adapting stimulus desensitizes multiple mechanisms in proportion to their respective sensitivities to it, thus tending to make them equivalent in sensitivity at the adapting frequency; for if, as a result of this adaptation-induced vertical shifting, the threshold functions of the two mechanisms intersect at any (adapting) frequency below $150 \mathrm{~Hz}$, then the frequency-dependent mechanism, because of its marked negative slope, will be the more sensitive of the two to a $150-\mathrm{Hz}$ test stimulus. An action spectrum obtained with respect to this test stimulus should therefore reflect the degree to which adapting stimuli of different frequencies activate the frequencydependent channel.

It is important to note that the $150-\mathrm{Hz}$ action spectrum has a salient high-frequency branch even in the case of Subject K, whose unadapted threshold function shows no such branch. Within the context of the two-channel model, this implies that Subject K, like the other subjects, has a frequency-dependent mechanism, but that it is hidden in the unadapted state, becoming manifest only in the action spectrum experiment, when adapting stimuli desensitize the frequency-independent system that had earlier concealed it. Thus, our data give no support to the notion that some subjects lack the frequency-dependent mechanism. The question of why evidence of this channel should appear in the unadapted threshold function of some subjects (Verrillo \& Ecker, 1977) but not others (Barlow, 1987) will be taken up in the General Discussion.

The two-channel model has difficulty, however, in explaining why the $150-\mathrm{Hz}$ action spectrum is shallow at low frequencies. This relatively flat branch resembles the 


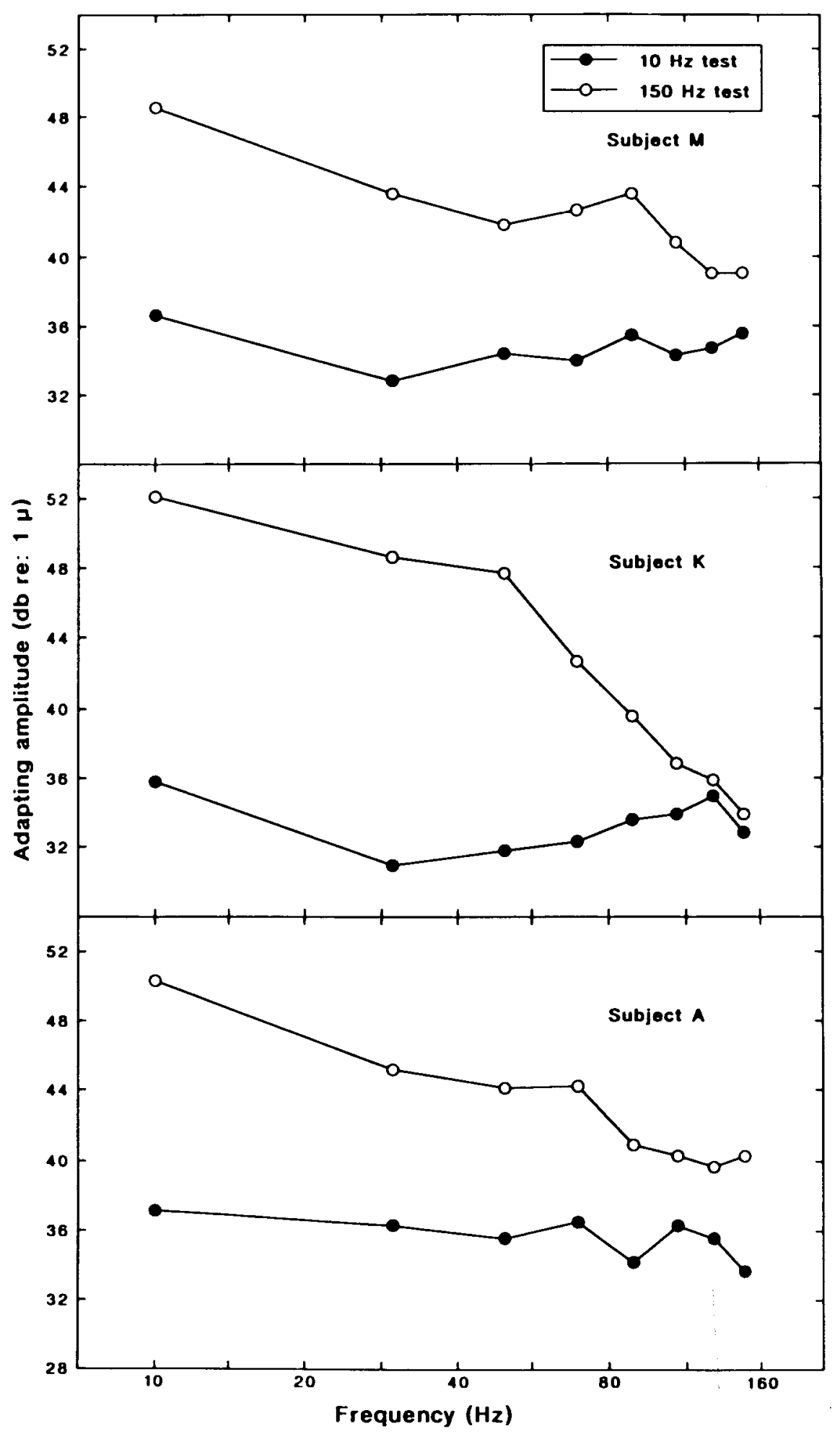

Figure 4. Action spectra of vibrotactile adaptation. Ordinate values represent the amplitudes (re: 1 micron peak displacement) needed, at each of the adapting frequencies plotted on the abscissa, to elevate by $10 \mathrm{~dB}$ the threshold of vibration at either $10 \mathrm{~Hz}$ (filled symbots) or $150 \mathrm{~Hz}$ (open symbols). 
frequency-independent mechanism thought to underlie the $10-\mathrm{Hz}$ action spectrum; but how could this mechanism be the more sensitive of the two to a $150-\mathrm{Hz}$ test? One possible interpretation is that at high amplitudes, there is an interaction between mechanoreceptive channels, such that signals in the frequency-independent channel can raise the threshold of the frequency-dependent channel. Although no evidence of channel interaction has been found in previous studies of vibrotactile adaptation (Gescheider et al., 1979; Gescheider \& Verrillo, 1979; Hahn, 1968; Hollins et al., 1990; Verrillo \& Gescheider, 1977), all of these earlier studies were conducted on the hand; it is possible that mechanoreceptive channels are less strictly isolated in the trigeminal system. Further research will be needed to determine with certainty whether this is the case.

\section{GENERAL DISCUSSION}

Two major conclusions can be drawn from this series of experiments. First, vibrotactile adaptation, previous study of which was largely confined to the glabrous skin of the hand, has been shown to occur on the face as well. This extension of knowledge is not merely parametric, for the two tissues are qualitatively different in a number of ways: The tested region of the face is hairy, rather than glabrous; it is served by the trigeminal system rather than by spinal nerves; and there are major differences between the mechanoreceptor populations in the two regions, including the fact that Pacinian corpuscles are largely absent from the face. It follows that vibrotactile adaptation is a phenomenon of some generality.

Our second major conclusion is that at least two mechanoreceptive systems participate in vibrotactile sensibility on the face. The evidence for this statement is that (1) the action spectrum of vibrotactile adaptation for a $10-\mathrm{Hz}$ test stimulus lies well below, and is different in shape from, the analogous function for a $150-\mathrm{Hz}$ test stimulus; and (2) the latter action spectrum is divided by a sharp inflection into low- and high-frequency portions differing in slope. Neither argument by itself is compelling, but together they make a strong case for at least two facial vibrotactile mechanisms.

Our study suggests two other conclusions, but does not establish them beyond doubt. The first is that a seeming discrepancy between two earlier studies of vibrotactile sensitivity on the face (Barlow, 1987; Verrillo \& Ecker, 1977 ) is probably attributable, at least in part, to differences between subjects. Our data show that, even when tested with the same apparatus and procedure, different individuals may have either a smooth, monotonic function resembling that reported by Barlow (1987), or a twobranched function like that of Verrillo and Ecker (1977).

Why were the groups they tested so different? One likely factor is age: Barlow's (1987) subjects were young-19 on the average-but Verrillo and Ecker's (1977) subjects ranged in age from 48 to 74 , with a mean of 62. The data of the present study are consistent with this interpretation, for our oldest subject (M, age 43) gave results comparable to those reported by Verrillo and Ecker, while the youngest (Subject K, age 22) had thresholds resembling those reported by Barlow. Clearly, however, further research is needed to systematically determine the effect of age on facial vibration sensitivity.

If the low-frequency branch of Verrillo and Ecker's (1977) threshold function and the entire threshold function obtained by Barlow (1987) represent the same mechanism, then we may describe the difference in results between the two earlier studies by saying that in the older subjects, an additional mechanism is revealed at high frequencies. This may result either from a decrease in threshold of the frequency-dependent mechanism, or an increase in threshold of the frequency-independent mechanism, with advancing age. By analogy with what is known about the effect of age on Pacinian sensitivity (Frisina \& Gescheider, 1977; Verrillo, 1977, 1979), we are inclined to favor the latter alternative, but the fact that Verrillo and Ecker's (1977) thresholds are substantially lower at all frequencies than those reported by Barlow (1987) leaves the issue unsettled. The small number of observers in the present study likewise precludes a resolution of this question at the present time.

A final possibility raised by the present study is that of interaction between channels, within the context of vibrotactile adaptation on the face. The fact that the $150-\mathrm{Hz}$ test action spectrum includes a low-frequency branch resembling in shape the $10-\mathrm{Hz}$ test action spectrum, but lying at least $8 \mathrm{~dB}$ above the latter function, suggests such interaction. Our data are consistent with the possibility that, at low and moderate sensation levels, vibrotactile adaptation occurs in complete independence within each channel, but that at high intensities of the adapting stimulus, signals in the frequency-independent channel produce additional elevation of the threshold of the frequencydependent mechanism. Such interaction between channels is absent in the hand (Gescheider et al., 1979; Gescheider \& Verrillo, 1979; Hahn, 1968; Hollins et al., 1990; Verrillo \& Gescheider, 1977), but the rules governing vibrotactile adaptation may be different on the face.

Correlation of the psychophysical mechanisms responsible for vibration sensitivity on the face, with specific anatomical structures in the skin, is problematic. The threshold function and action spectrum of what we have called the frequency-independent mechanism resemble in shape the NP I mechanism of Bolanowski et al. (1988); however, that mechanism, defined on the glabrous skin of the hand, is believed to reflect the activity of Meissner corpuscles, which do not occur in hairy skin. Merzenich and Harrington (1969) have discovered a psychophysical mechanism resembling NP I on the hairy skin of the forearm, and they have presented evidence that it depends on the activity of sensory nerve endings associated with hairs; but there are at present insufficient physiological data to allow this correlation to be extended to the face.

There is even less basis for assigning the frequencydependent mechanism studied here to a particular type of afferent. Verrillo and Ecker (1977) suggested that the 
high-frequency branch of their threshold function might be attributable to Golgi-Mazzoni corpuscles, a type of rapidly adapting mechanoreceptor found in perioral tissues (Sakada, 1971). This correlation must be regarded as tentative, however, in view of the parametric similarity of the frequency-dependent mechanism to the NP II channel of Bolanowski et al. (1988), which, according to evidence presented by those authors, is mediated by slowly adapting type II afferents. Physiological "tuning curves" of different classes of facial afferents are needed to resolve these questions.

In summary, in the present study we have established the generality of vibrotactile adaptation, and have used it to demonstrate that vibration sensibility on the face differs in a number of important ways from that on the hand. Because of the unique role of perioral skin in eating and speaking - two activities that involve considerable vibration of tissue-it is likely that continued research on basic mechanisms of facial vibrotaction will yield information of both theoretical and clinical significance.

\section{REFERENCES}

BARLow, S. M. (1987). Mechanical frequency detection thresholds in the human face. Experimental Neurology, 96, 253-261.

Bolanowski, S. J., JR., Gescheider, G. A., Verrillo, R. T., \& Checkosky, C. M. (1988). Four channels mediate the mechanical aspects of touch. Joumal of the Acoustical Society of America, 84, 1680-1694.

CHubBuck, J. G. (1966). Small-motion biological stimulator. APL Technical Digest, May-June, 18-23.

COHEN, L. H., \& LINDLEY, S. B. (1938). Studies in vibratory sensibility. American Journal of Psychology, 51, 44-63.

Darian-SMITH, I. (1973). The trigeminal system. In A. Iggo (Ed.), Handbook of sensory physiology: Vol. II. Somatosensory system (pp. 271-314). Berlin: Springer.

Frisina, R. D., \& Gescheider, G. A. (1977). Comparison of child and adult vibrotactile thresholds as a function of frequency and duration. Perception \& Psychophysics, 22, 100-103.

Gescheider, G. A. (1976). Evidence in support of the duplex theory of mechanoreception. Sensory Processes, 1, 68-76.

Gescheider, G. A., Frisina, R. D., \& Verrillo, R. T. (1979). Selective adaptation of vibrotactile thresholds. Sensory Processes, 3, 37-48.

Gescheider, G. A., \& Niblette, R. K. (1967). Cross-modality masking for touch and hearing. Journal of Experimental Psychology, 74, 313-320.
Gescheider, G. A., \& VerRllo, R. T. (1979). Vibrotactile frequency characteristics as determined by adaptation and masking procedures. In D. R. Kenshalo (Ed.), Sensory functions of the skin of humans (pp. 183-205). New York: Plenum.

HaHN, J. F. (1968). Low-frequency vibrotactile adaptation. Journal of Experimental Psychology, 78, 655-659.

Halata, Z., Munger, B. L. (1983). The sensory innervation of primate facial skin: II. Vermilion border and mucosa of lip. Brain Research Reviews, 5, 81-107.

Hollins, M., Goble, A. K., Whitsel, B. L., \& TOMmerdahl, M. (1990). Time course and action spectrum of vibrotactile adaptation. Somatosensory \& Motor Research, 7, 205-221.

JoHansson, R. S., \& Olsson, K. A. (1976). Micro-electrode recording from human oral mechanoreceptors. Brain Research, 118, 307-311.

Johansson, R. S., Trulsson, M., Olsson, K. A., \& Westberg, K.-G. (1988). Mechanoreceptor activity from the human face and oral mucosa. Experimental Brain Research, 72, 204-208.

Merzenich, M. M., \& Harrington, T. (1969). The sense of fluttervibration evoked by stimulation of the hairy skin of primates: Comparison of human sensory capacity with the responses of mechanoreceptive afferents innervating the hairy skin of monkeys. Experimental Brain Research, 9, 236-260.

Munger, B. L., \& Halata, Z. (1983). The sensory innervation of primate facial skin: I. Hairy skin. Brain Research Reviews, 5, 45-80.

SAKADA, S. (1971). Response of Golgi-Mazzoni corpuscles in the cat periostea to mechanical stimuli. In R. Dubner \& Y. Kawamura (Eds.), Oral-facial sensory and motor mechanisms (pp. 105-122). New York: Appleton-Century-Crofts.

VERRILLO, R. T. (1963). Effect of contactor area on the vibrotactile threshold. Journal of the Acoustical Society of America, 35, 1962-1966.

VeRrilLo, R. T. (1966). Vibrotactile sensitivity and the frequency response of the Pacinian corpuscle. Psychonomic Science, 4, 135-136.

VERRILLO, R. T. (1968). A duplex mechanism of mechanoreception. In D. R. Kenshalo (Ed.), The skin senses (pp. 139-159). Springfield, IL: Thomas.

Verrillo, R. T. (1977). Comparison of child and adult vibrotactile thresholds. Bulletin of the Psychonomic Society, 9, 197-200.

VERRILLO, R. T. (1979). Change in vibrotactile thresholds as a function of age. Sensory Processes, 3, 49-59.

VerRILLo, R. T., \& ECKER, A. D. (1977). Effects of root or nerve destruction on vibrotactile sensitivity in trigeminal neuralgia. Pain, 3, 239-255.

VerRillo, R. T., \& Gescheider, G. A. (1977). Effect of prior stimulation on vibrotactile thresholds. Sensory Processes, 1, 292-300.

WEDELL, C. H., \& Cummings, S. B., JR. (1938). Fatigue of the vibratory sense. Journal of Experimental Psychology, 22, 429-438.

(Manuscript received March 16, 1990; revision accepted for publication July 10, 1990.) 